\title{
Hydrodynamic simulations of outburst events in accretion discs in close binaries
}

\author{
Giuseppe Lanzafame* \\ INAF - Osservatorio Astrofisico di Catania, Via S. Sofia 78, I-95123 Catania, Italy \\ E-mail: glanzafamedoact.inaf.it
}

\section{Vincenzo Costa}

Dipartimento di Metodologie Fisiche e Chimiche per l'Ingegneria, Universitá di Catania, Via A. Doria 6, I-95125 Catania, Italy

E-mail: vcosta@oact.inaf.it

\section{Gaetano Belvedere}

Dipartimento di Fisica e Astronomia, Sez. Astrofisica, Universitá di Catania, Via S. Sofia 78, I-95123 Catania, Italy

E-mail: gbelvedere@oact.inaf.it

In this work, the role of both inflow kinematic at the inner Lagrangian point L1 as initial boundary condition, and gas compressibility, and physical turbulent viscosity, on accretion disc's dynamics and structure in a Close Binary (CB), are investigated via simulations of 3D SPH stationary accretion disc models. Physical viscosity hampers the gas dynamics (rarefaction or compression), supporting the accretion disc development inside the primary gravitational potential well in a CB system, even for low compressibility modelling. Currently, the turbulent Shakura-Sunyaev parameter $\alpha \simeq 0.1$ is widely adopted for these structures. Such investigation is here carried out in both high and low compressibility regimes, with the aim of evaluating, in a compressibility-viscosity graph, the most suitable domains where physical conditions allow a well-bound disc development as a function of mass transfer kinematic conditions. A selection of the adopted $\alpha$ physical turbulent viscosity parameter ( $\alpha \leq 1$ according to the well-known Shakura-Sunjaev formulation) has been considered, whilst the adopted polytropic index $\gamma$ is chosen in the range between $1 \div 5 / 3$. Results show that domains exist where physical turbulent viscosity supports the accretion disc formation. In such domains, the lower the gas compressibility, the higher the physical viscosity requested. A role played by the injection kinematics at the inner Lagrangian point L1 is also found. Conclusions as far as dwarf novae outbursts are concerned, induced by mass transfer rate variations, are also reported. Considerations as far as periodicities in an accretion disc are also reported.

VII Microquasar Workshop: Microquasars and Beyond September 1-5 2008

Foca, Izmir, Turkey

${ }^{*}$ Speaker. 


\section{Introduction}

In [9] it is shown that physical viscosity supports the accretion disc development inside the primary gravitational potential well, despite the low compressibility conditions adopted. Inviscid simulations in low compressibility conditions (e.g. [12, 8]) do not produce well-bound accretion discs within the primary's gravitational potential well by the fact that blobs of gas escape from the disc outer edge because the ejection rate is comparable to the injection rate from the inner Lagrangian point L1. High compressibility viscous gas dynamics in accretion discs was investigated in [5]. In a high compressibility modelling, accretion discs would form anyway, even in physically inviscid conditions. The physical, turbulent viscosity modifies the disc geometry and thermodynamics. In fact, highly compressible viscous disc models are thinner, hotter and more radially extended. Moreover, in spite of a degradation viscous effect, spiral structures and shocks are more prominent.

Physical viscosity works where the particle mutual velocity (and separation) changes in time, namely when a mutual acceleration exists, contrasting gas dynamics (rarefaction or compression) where particle velocity gradients are significant. This means that physical viscosity plays a relevant role mainly in the radial transport, while it has little influence on the tangential dynamics. It converts mechanical energy into thermal energy (heating the disc), supporting the development of well-bound accretion discs inside the primary potential well, even in spite of a hypothetical low compressibility gas ([9]). It reduces the disc thickness and increases the accretion rate onto the accreting star, but, and this is the most important thing, it hampers the repulsive pressure forces among contiguous fluid elements supporting the accretion disc in developing a well-bound consistency.

A grid of SPH disc models is here produced, with the aim of detecting, in the compressibilityviscosity space, boundaries separating domains where the disc development is supported from domains where it is not. The injection velocity in L1 could also have a role. Therefore, in order to stress this idea in this paper, according to fixed kinematic injection conditions at the L1 point as an initial boundary condition, several polytropic indexes $\gamma$ have been adopted, identifying, for each of them, the boundary lower limit of Shakura-Sunyaev parameter $\alpha[13,14]$ able to provide a sufficient particle concentration to define a well-bound accretion disc into the primary's gravitational potential well. The polytropic index $\gamma$, introduced in the state equation, $p=(\gamma-1) \rho \varepsilon$, where $\varepsilon$ is the thermal energy per unit mass, has the meaning of a numerical parameter whose value lies in the range between 1 and 5/3, in principle. All simulations are carried out ensuring a stationary final configuration where the rate of injected particles is balanced by the rates of accreted and ejected particles and the number of disc particles is statistically conserved. The same binary system parameters - stellar masses and their separation - have been adopted throughout.

In order to build up a "well-bound" accretion disc in inviscid conditions, the ejection rate at the disc's outer edge must be at least two or three times lower than the accretion rate at the disc's inner edge. Whenever this condition is fulfilled, the disc's outer edge, as well as the whole disc, does not "evaporate" because of high pressure forces. Therefore, low compressibility gases are more easily sensitive to evaporative effects of blobs of gas at the disc's outer edge itself, towards the empty external space, if the gravitational field is not able to keep disc gas in the gravitational potential well at the disc's outer edge. The physical turbulent viscosity contributes to the gas confinement 
in the gravitational potential well, starting from a lower threshold to be defined, which is the main aim of this work. Of course, the inclusion of physical viscosity terms supports the radial transport of gas towards the centre of the gravitational potential well.

The assignation of $\alpha \simeq 0.1$ is quite common $([3,13,11,16,10])$ as an order of magnitude for thin accretion discs in close binaries.

In this paper, the viscous force contribution is represented by the divergence of the symmetric viscous stress tensor in the Navier-Stokes equation. A symmetric combination of the symmetric shear tensor multiplied by the particle velocity has been added to the energy equation as a viscous heating contribution. The bulk physical viscosity contribution has not been considered for the sake of simplicity. The SPH formulation of viscous contributions in Navier-Stokes and energy equations has been developed by $[1,2]$. They adopted a stress tensor whose shear component is proportional to the Shakura-Sunjaev parameter $\alpha$ multiplied by the gas pressure and excluding any artificial viscosity contribution.

The adopted SPH scheme, as with any finite difference numerical method, or any finite volume numerical method, is affected by the adopted spatial resolution length $h$. The radial transport is also affected by the SPH particle resolution length $h$ since too small $h$ values prevent the radial transport. Instead, high $h$ values produce a too high radial transport of matter towards the centre of the gravitational potential well. The choice of a suitable fixed resolution length $h$ of the order of $10^{-2}$ of the whole linear spatial integration domain where particles move. $h=0.005$ is one of the best compromise solutions in our modelling. A high $h$ value ensures a high particle overlapping (interpolation) but at the same time it produces a high particle repulsion rate due to pressure forces especially in low compressibility regimes at the disc edges. This further prevents disc binding into the primary's gravitational potential well. On the contrary, a too low $h$ resolution length compromises any fluid dynamic behaviour and shock handling.

\section{Modelling parameters and results}

We carried out our simulations until we achieved full stationary configurations. This means that particles injected into the primary's gravitational potential well are statistically balanced by particles accreted onto the primary and by particles ejected from the disc's outer edge. Critical boundary conditions for the development of a well-defined accretion disc, for each pairs of $(\gamma, \alpha)$ values, were pursued, ensuring a minimum number of particle neighbours of the order of 15 at least for each SPH particle.

The characteristics of the binary system are determined by the masses of the two stars and their separation. We chose to model a system in which the mass $M_{1}$ of the primary compact star and the mass $M_{2}$ of the secondary normal star are equal to one $M_{\odot}$ and the distance between their centres is $d_{12}=10^{6} \mathrm{Km}$. The injection gas velocity in L1 is fixed at $v_{i n j} \simeq 13 \mathrm{Km} \mathrm{s}^{-1}$, at $v_{i n j} \simeq 50 \mathrm{Km} / \mathrm{s}$ and at $v_{i n j} \simeq 130 \mathrm{Km} \mathrm{s}^{-1}$ for our three groups of models while the injection gas temperature in L1 is fixed at $T_{\circ}=10^{4} \mathrm{~K}$. Such a temperature value is slightly higher than the value corresponding to a normal $1 M_{\odot}$ star because, even as a first approximation, the radiative heating of the secondary surface due to the disc enlightening is taken into account. Gas compressibility is fixed by the adiabatic index $\gamma$.

In order to make our equations dimensionless, we adopted the following normalization factors: $M=M_{1}+M_{2}$ for masses, $d_{12}=10^{11} \mathrm{~cm}$ for lengths, $v_{\circ}=\left(G\left(M_{1}+M_{2}\right) / d_{12}\right)^{1 / 2}$ for speeds, so 
that the orbital period is normalized to $2 \pi, \rho_{\circ}=10^{-9} \mathrm{~g} \mathrm{~cm}^{-3}$ for the density, $p_{\circ}=\rho_{\circ} v_{\circ}^{2} d y n \mathrm{~cm}^{-2}$ for pressure, $v_{\circ}^{2}$ for thermal energy per unit mass and $T_{\circ}=(\gamma-1) v_{\circ}^{2} m_{p} K_{B}^{-1}$ for temperature, where $m_{p}$ is the proton mass and $K_{B}$ is the Boltzman constant. The adopted kernel width in the SPH interpolations is $h=0.005$ and the geometrical domain, including moving disc particles, is a sphere of radius 0.6 centred on the primary. The inverse of the resolution length $1 / h=200$. This value represents, at least in $1 \mathrm{D}$, as a typical order of magnitude, the number of finite difference elements dividing any spatial integration domain in order to solve any system of partial differential equations in fluid-dynamics.

The rotating reference frame is centred on the compact primary and its rotational period equals the orbital one. We simulated the physical conditions at the inner and at the outer edges as follows:

a) Inner edge:

The free inflow condition is realized by eliminating particles flowing inside the sphere of radius $4 h$, centred on the primary. It follows that disc structure and dynamics are altered within a few $h$ values of the inner edge, adopting a fixed $h$ SPH code, but these alterations in disc behaviour are relatively small because they are counterbalanced by a higher particle concentration close to the inner edge in supersonic injection models.

b) Outer edge:

The injection of "new" particles from L1 towards the interior of the primary Roche Lobe is simulated by generating them in fixed points, called "injectors", symmetrically placed within an angle having L1 as a vertex and an aperture of $\sim 57^{\circ}$. The initial injection particle velocity is radial compared to L1. In order to simulate a constant and smooth gas injection, a "new" particle is generated in the injectors whenever "old" particles leave an injector free, inside a small sphere of radius $h$, centred on the injector itself.

The formulation adopted for the 3D SPH accretion disc model including physical viscosity is the well-known Shakura \& Sunyaev $([14,15]) \alpha$ parametrization: $v=\alpha c_{s} H$, where $0 \leq \alpha \leq 1$ is the Shakura-Sunyaev parameter and $H=r_{x y} c_{s} /\left(M_{1} / r_{x y}\right)^{1 / 2}$ is a dimensionless estimate of the standard disc thickness, where $r_{x y}=\left(X_{i}^{2}+Y_{i}^{2}\right)^{1 / 2}$ is the cylindrical radial coordinate of the ith particle.

The mass transfer injection rates from L1 $\dot{M}_{i n j}$, as well as the accretion rates $\dot{M}_{a c c}$ and the ejection rates $\dot{M}_{e j e}$ for $v_{i n j}=13 \mathrm{Km} / \mathrm{s}, v_{i n j}=50 \mathrm{Km} / \mathrm{s}$ and $v_{i n j}=130 \mathrm{Km} / \mathrm{s}$ at the $\mathrm{L} 1$ point, respectively, are reported in Table 1. $\rho_{\circ} h^{3} d_{12}^{2} v_{\circ}$, is the conversion factor from particle/time to $g s^{-1}$. Mass accretion and ejection rates, due to the Shakura-Sunyaev viscosity parameter $\alpha$ adopted, can be altered by $\sim 10 \% \div 20 \%$ for accretion rate and by a factor $3 \div 4$ for ejection rate. The higher $\alpha$, the lower the ejection rate (the higher the accretion rate). Instead, the lower the gas compressibility (the higher $\gamma$ ), the higher the ejection rate (the lower the accretion rate), although the main characteristic, governing both the accretion and the ejection rate, is the injection rate in L1.

An estimate of the diffusion characteristic time, mainly responsible for the disc's radial spread, yields $t_{\text {diff }} \sim R_{\text {disc }}^{2} / \nu \sim 0.4 \div 18.1$ as the lower and the higher values, respectively, from our models, considering $t_{\text {diff }} \sim R_{\text {disc }}^{2} /\left(\alpha c_{s} H\right)$, where $H \sim 0.1$ and $R_{\text {disc }} \sim 0.25 \div 0.3$. Such evaluation also takes into account the artificial viscosity contribution which, in our results, is comparable to the physical viscosity contribution for $\alpha \sim$ hundredths, taking into account of a sound velocity which is within the range $\simeq 4 \div 8$ in the disc bulk in non-dimensional units. The two opposite values, being lower than $t=25 \simeq 3.98$ orbital periods, ensure that the pursued steady state is statistically 


\begin{tabular}{cccc}
$\dot{M}_{i n j}\left[g \cdot s^{-1}\right]$ & $\dot{M}_{a c c}\left[g \cdot s^{-1}\right]$ & $\dot{M}_{e j e}\left[g \cdot s^{-1}\right]$ & $v_{i n j}\left[\mathrm{Km} \cdot \mathrm{s}^{-1}\right]$ \\
\hline $2.3 \cdot 10^{16}$ & $1.6 \cdot 10^{16}$ & $7.0 \cdot 10^{15}$ & 13 \\
$3.6 \cdot 10^{16}$ & $2.4 \cdot 10^{16}$ & $1.2 \cdot 10^{16}$ & 50 \\
$6.5 \cdot 10^{16}$ & $3.5 \cdot 10^{16}$ & $3.0 \cdot 10^{16}$ & 130
\end{tabular}

Table 1: Injection, accretion and ejection rates relative to the adopted injetion velocity $v_{i n j}$ at L1 point. Values are indicative whichever is the adopted $(\gamma, \alpha)$ pair.

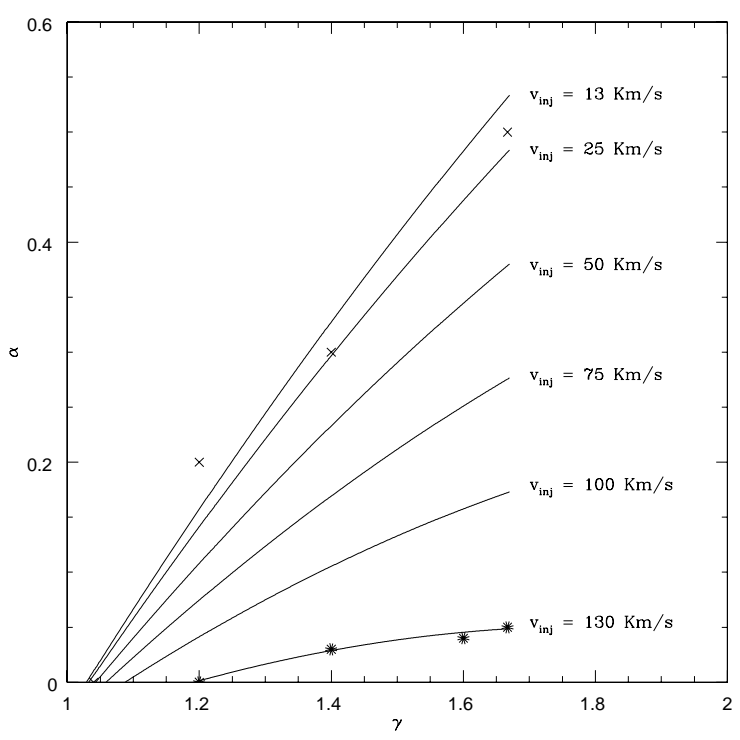

Figure 1: $(\gamma, \alpha)$ plot of boundaries separating domains where physical turbulent viscosity allows the development of a statistically defined accretion disc (above each boundary) from domains where it is not (below each boundary). Injection velocities $v_{i n j}$ at the inner Lagrangian point $\mathrm{L} 1$, are also reported for each boundary. Crosses and asterisks refer to $(\gamma, \alpha)$ pairs used for defining the outermost boundaries.

fulfilled.

Fig. 1 shows a $(\gamma, \alpha)$ (compressibility versus physical turbulent viscosity) diagram where boundaries, separating domains where the disc development is supported (domain above each boundary) from domains where it is not (domain below each boundary), are reported, performing parabolic best fits, taking into account such results.

For disc models whose $\alpha>\alpha_{b}$, the physical, turbulent viscosity is able to develop a wellbound accretion disc in the primary's gravitational potential well, while disc models whose $\alpha<\alpha_{b}$ do not.

These results show that not only the pair of $(\gamma, \alpha)$ values are important in the development of a well-bound accretion disc modelling in the primary star gravitational potential well, but also that the injection velocity at the $\mathrm{L} 1$ point has a relevant role. 


\section{Discussion}

These results have a direct consequence for the dwarf novae outburst modelling. As an example, we can consider a well-bound accretion disc in the primary's gravitational potential well in a stationary condition fulfilled for a high mass transfer rate from L1 point (e.g. a point in the diagram above the parabolic boundary for $v_{i n j}=130 \mathrm{Km} / \mathrm{s}$ ). The pair $\gamma=7 / 5$ and $\alpha=0.1$ corresponds to this condition. According to the fixed $(\gamma, \alpha)$ adopted, a large disc radius is quite a natural consequence due to the high mass transfer from L1 point. This is our starting disc configuration. The disc is shortly reduced and (maybe) dissolved for the same pair $(\gamma, \alpha)$ (especially for high values of $\gamma>1.2)$ if the mass transfer rate decreases as a consequence of the reduction in the injection velocity in L1. In this case the pair of $(\gamma, \alpha)$ values identifies the same point below the new parabolic boundary identified by the reduced $v_{i n j}$. Disc evolution is shown in Fig. 2 regarding the transition between opposite stationary configurations from $v_{i n j}=130 \mathrm{Km} / \mathrm{s}$ to $v_{i n j}=13 \mathrm{Km} / \mathrm{s}$ in L1 and vice-versa. According to these results, in high compressibility regimes, dwarf novae outbursts temporarily represent phases of a well-bound accretion disc when its radius increases as a consequence of the higher mass transfer rate from L1 point. Alternatively they could represent phases where a low compressibility disc achieves a consistency when $\dot{M}_{a c c} \sim 10^{17} \mathrm{gs}^{-1}$. Instead, in high compressibility regimes, quiescent phases represent low rate mass transfer phases where the accretion disc reaches its minimum radial extension as a consequence of the reduced mass transfer rate from L1. Alternatively, in low compressibility regimes, they could represent even vanishing disc phases when the disc radius not only decreases, but also dissolves when $\dot{M}_{a c c} \sim 10^{16} g s^{-1}$. A higher accretion rate onto the primary star involves a higher emission flux (especially in the X-ray close to the primary compact star) by conversion of mechanical energy into heat. Before the beginning or after the end of the transition phase the accretion disc is in a steady state and a statistical balance exists among the injection rate from L1, the ejection rate from the disc's outer edge and the accretion rate onto the primary compact star. When the transitional phase begins from a higher injection rate, from L1 to a lower injection rate or vice-versa, the disc bulk gradually evolves, adjusting its structure to the modified outer edge injection conditions at the L1 point. Such disc outer edge injection conditions involve not only the mass transfer rate itself, but also the angular momentum transport from L1. A higher injection velocity statistically involves a higher injected angular momentum from L1, because of a higher velocity component, perpendicular to the axis joining the two stellar components. As a consequence, the higher the injection velocity, the higher the disc radius when a steady state is fulfilled. The transitional length between the opposite stationary disc configurations (bottom to top of the figure) is $\sim 10$ and $\sim 5$ orbital periods, respectively. Such values agree, as an order of magnitude, with the outburst duration, as shown in [4], where, among several hypotheses, a periodical modulation of the mass transfer rate is also taken into account as responsible for SU UMa, OY Car, Z Cha and SS Cyg-like systems periodical variation of systemic velocity.

Conclusions are not so evident if $\gamma<1$.1. In fact, in permanent high compressibility conditions this phenomenology, explaining dwarf novae outbursts, should not be so stressed, although periodical quiescent-active phases are still significant. These conclusions are due to the identification of domains in the compressibility-turbulent viscosity $(\gamma, \alpha)$ diagram, where the accretion disc consistency is allowed, according to the kinematic conditions in L1. Results in this paper agree with 


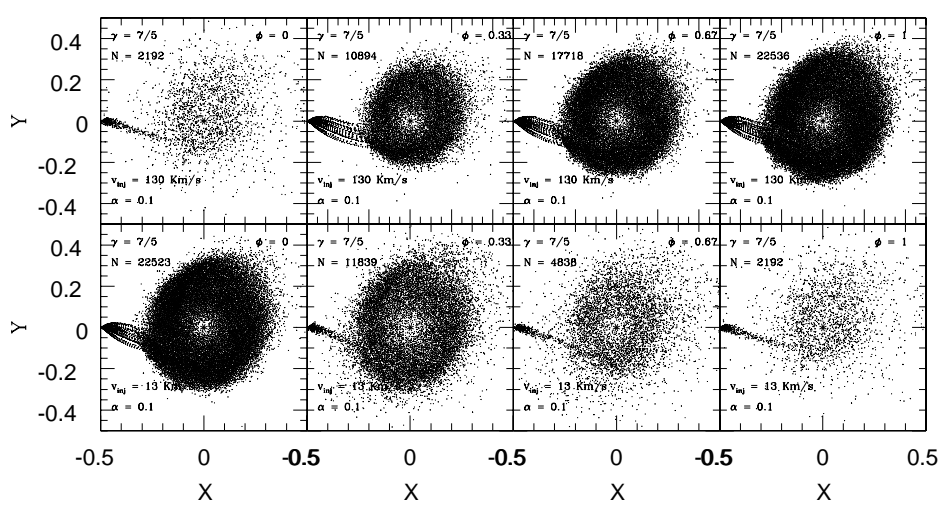

Figure 2: XY plots of transitional phase active-toquiet (top 4 plots) and vice-versa (bottom 4 plots) of accretion disc. The $\gamma, \alpha$ ) pair, the $v_{i n j}$, as well as the phase $\phi$ are also reported.

observational evidence concerning outbursts and show the role of compressibility, physical turbulent viscosity and mass transfer kinematics on accretion disc structure, dynamics and energetics. This paper, together with Lanzafame's [5] results, shows that any numerical viscosity parameter is theoretically possible in order to get well-bound accretion discs in high compressibility regimes. Instead, both high viscosity values, together with high mass transfer rates from th L1 point, support the disc binding into the primary's gravitational potential well according to the $(\gamma, \alpha)$ diagram of Fig. 1 in low compressibility regimes.

The final comment concerns the reason why a physically viscous disc gas that is in a low compressibility regime $(\gamma \geq 1.1)$ better enhances an outburst event than a high compressibility one $(\gamma<1.1)$. As shown in $[12,8,5]$ the disc radial extension and its binding into the primary's gravitational potential well is not very sensitive to the mass transfer rate kinematical conditions in L1 when $\gamma<1$.1. Injection kinematical conditions in L1 can only affect the development of spiral shaped structures when high mass transfer rates conditions occur in L1, especially in inviscid conditions. The total number of disc particles in stationary condition differs by a factor $4 \div 5$ between the two opposite mass transfer conditions considered in such high compressibility regimes. Instead, a relevant difference between the two opposite stationary structures (quiescent - outburst) exists, in particular regarding disc's radial extension and disc's local densities when $\gamma \geq 1.1$. Even the total number of disc particles in stationary condition differs by a factor of $\sim 10$ between the two opposite mass transfer conditions considered when $\gamma \geq 1$.1. This implies that in low compressibility regimes not only does the disc's radial extension effectively vary, but also the disc's local opacity and the accretion rate onto the primary star. Therefore, while in high compressibility the transitional phase between quiescence and outburst (and vice-versa) seems more gradual, it is much more violent in a low compressibility regime. Of course, this does not preclude accretion discs in close binaries from effectively being in a high compressibility condition. 


\section{References}

[1] Flebbe, O., Münzel, H., Riffert, H. \& Herold, H., 1992, Mem. S.A.It, 65, 1049.

[2] Flebbe, O., Münzel, H., Herold, H., Riffert \& H., Ruder, H., 1994, ApJ, 431, 754.

[3] Hassal, B.J.M., Pringle, J.E., Verbunt, F., MNRAS, 215, 353.

[4] Honey, W.B., Charles, P.A., Whitehurst, R., Barret, P.E., Smale, A.P. 1988, MNRAS, 31, 1

[5] Lanzafame, G., 2003, A\&A, 403, 593.

[6] Lanzafame, G., 2008a, "The Role of Physical Viscosity in Accretion Disc Dynamics in Close Binaries and AGN", in "Numerical Modeling of Space Plasma Flows: Astronum 2007", 10-15 June 2007, Paris, France, N. V. Pogorelov, E. Audit, and G. P. Zank (eds), ASP Conference Series, 385, p.115.

[7] Lanzafame, G., 2008b, PASJ, 60, 259.

[8] Lanzafame, G., Belvedere G., Molteni D., 1992, MNRAS, 258, 152.

[9] Lanzafame, G., Belvedere G. \& Molteni, D., 2006, A\&A, 453, 1027.

[10] Lasota, J.P., 2001, New Astr. Rev., 45, 449.

[11] Livio, M., Verbunt, F., 1988, MNRAS, 232, 1P.

[12] Molteni, D., Belvedere, G. Lanzafame, G., 1991, MNRAS, 249, 748.

[13] Pringle, J.E., Verbunt, F., Wade, R.A., 1986, MNRAS, 221, 169.

[14] Shakura, N.I., 1972, Astron. Zh., 49, 921. (English tr.: 1973, Sov. Astron., 16, 756).

[15] Shakura, N.I. \& Sunyaev, R.A., 1973, A\&A, 24, 337.

[16] Verbunt, F., Rappaport, S., 1988, ApJ, 332, 193. 